\title{
XXIV. On the mean free path of the molecules
}

\section{N.D.C. Hodges}

To cite this article: N.D.C. Hodges (1880) XXIV. On the mean free path of the molecules, Philosophical Magazine Series 5, 9:55, 177-180, DOI: 10.1080/14786448008626823

To link to this article: http://dx.doi.org/10.1080/14786448008626823

曲 Published online: 08 Jun 2010.

Submit your article to this journal

Џ Article views: 2

Q View related articles $₫$ 
is that which leads to Fechner's law. It is possible that this law may not be accurately and rigorously true; that would only be to say that our fundamental hypotheses err in the same manner, which is not at all improbable. But until experiments of a far more careful and extensive character than any yet made come to decide the question, we may take Fechner's law to be highly probable.

This law will always probably be best led up to by the physiological reasoning commonly employed. But I have thought it not useless to examine the consequences of the various elementary assumptions as to the measure of the sensation of sound, from a different point of view.

P.S.-Since the above was written, my attention has been drawn to an experimental verification of Fechner's law, for the sounds produced by falling woights (Carl Nörr, Zeitschrift für Biologie, 1879, p. 297).

XXIV. On the Mean free Path of the Molecules. By N. D. C. HodGes*.

THE free path of a molecule is dependent on the amount 1 of obstruction it meets with, or the density of the medium. O. E. Meyer gives for the mean free path (on page 308 of his Kinetische Theorie der Gase), $\mathrm{L}=\frac{1}{\pi \sqrt{2} \mathrm{~N} \zeta^{2}}$. Here $\mathrm{N}$ is the number of molecules in the unit volume.

I consider the length of path in a medium of variable density. At the surface of a liquid, if there is no sharp transition from the liquid to the gaseous state, we shall have a succession of layers of less and less dense vapours, from where there is liquid to the surrounding atmosphere. The layers $\mathrm{V}$ are what I refer to. The depth of these vapours is, of course, much magnified.

I propose to find the pressure upon the particle $p$, when the surface of the liquid is plane and when it is spherical. Taking the molecules moving with any definite velocity, they will reach $p$ and give it an impulse when they are at a distance from $p$ less than their mean free path. Now the molecules from below come from denser layers than those from above. A greater number will come from below than from above; there will be a tendency to drive $p$ upward. To find this tendency, we must find how much denser the lower layers, from

* Communicated by the Author. 
which molecules impinging on $p$ come, are than those above, from which particles come to $p$.

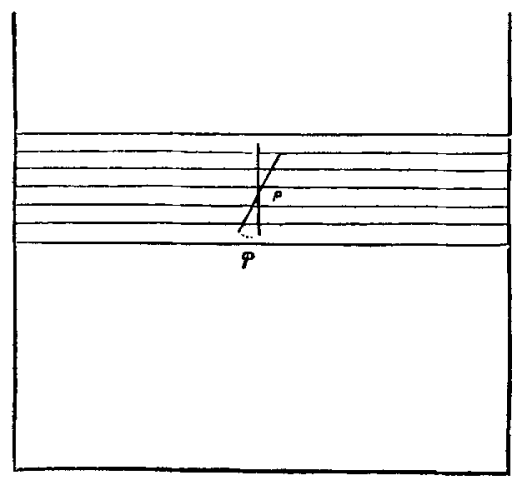

If $\delta \rho$ is the obstruction met by a molecule in passing vertically upward through a single layer, $\frac{\delta \rho}{\cos \phi}$ will be that met in a direction at an angle $\phi$ with the vertical. The integral $\int_{\rho_{0}}^{\rho_{1}} \frac{d \rho}{\cos \phi}$ gives the obstruction met with by the molecule from one end to the other of its path. This integral must be constant; for the length of path is independent of the direction. As the differential of the obstruction is the same as the differential of the density,

$$
\frac{1}{\cos \phi} \int_{\rho_{0}}^{\rho_{1}} d \rho=\frac{\rho_{1}-\rho_{0}}{\cos \phi}
$$

where $\rho_{0}$ is the density at the point $p$, and $\rho_{1}$ that at the other end of the path. As $\frac{\rho_{1}-\rho_{0}}{\cos \phi}=k$, the numerator $\rho_{1}-\rho_{0}$ must be proportional to $\cos \phi$. The pressure on $p$ is proportional to this difference, and the resultant component in the upward direction to $\cos ^{2} \phi$.

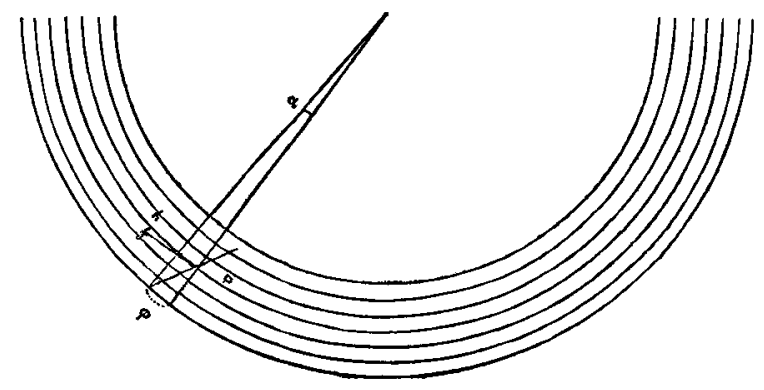


When the surface is spherical, each element of the path

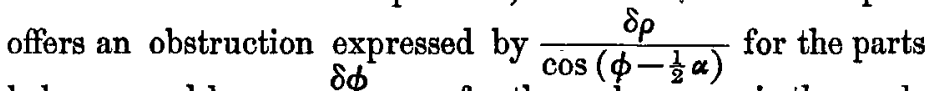
below $p$, and by $\frac{\delta \phi}{\cos \left(\phi+\frac{1}{2} \alpha\right)}$ for those above. $\alpha$ is the angle between the radius of curvature at the point $p$ and that to the other end of the path; $\phi-\frac{1}{2} \alpha$ is the mean value of the angle between the direction of path and the normals to the surfaces of equal density for the parts below $p$, and $\phi+\frac{1}{2} \alpha$ the corresponding angle for those above $p$.

Integrating,

$$
\frac{\rho_{1}^{\prime}-\rho_{0}^{\prime}}{\cos \left(\phi-\frac{1}{2} \alpha\right)}=k=\frac{\rho_{0}^{\prime}-\rho_{2}^{\prime}}{\cos \left(\phi+\frac{1}{2} \alpha\right)} \text {. }
$$

This shows that, whereas the difference in density above and below was the same for a plane surface, in the case of a spherical surface the pressure from below is greater, and that upward less. Or the pressure from below is greater and that from above greater. This must cause a greater density at $p$.

The tendency of a particle to move from the liquid into the surrounding atmosphere is due to the difference in density of the liquid and of its vapour. For small changes in the density, the change in this tendency may be assumed as proportional to the change of density. It must be found what change of density takes place at $p$. As the change of density is due to an increase of pressure on $p$, this increase must be equal in all directions. So it is only necessary to consider one direction. Take the direction tangent to the curved surface at $p$. The increase in pressure is therefore proportional to the difference in density of the layer through $f$ and that through $h$, or to the length $f h$. It is evident that

$$
\frac{f h}{h}=\frac{\frac{1}{2} h}{r}
$$

where $h$ is the free path, and $r$ the radius of curvature of the surface. Hence we have

$$
\frac{\text { the change in tension }}{\text { the tension of vapour at plane surface }}=\frac{\frac{1}{2} h}{r} \text {. }
$$

Sir William Thomson has shown that the change in tension at a curved surface is equal to the pressure of a column of the vapour of the height to which the liquid would rise in a capillary tube of a diameter of twice the radius of curvature of the surface.

In a tube of diameter 1.294 millim. water rises to a height 
of 23.379 millim. The data for the calculation are :-

Weight of a litre of water-vapour at $100^{\circ} \mathrm{C} . \quad .80357 \mathrm{grm}$. ", " " mercury .. . . 13.579 grms. Tension of vapour of water at $20^{\circ} \mathrm{C} .{ }^{\circ} \cdot{ }^{\circ} \quad 18.495$ millims.

The height of a column of mercury equivalent to the column of water-vapour of height 23.379 millims., is

$$
\frac{.80357}{13 \cdot 579} \cdot 100 \cdot \frac{18 \cdot 495}{760} \cdot \frac{23 \cdot 379}{100} \text {. }
$$

The first factor is the fraction of a litre of mercury which a litre of water-vapour at $100^{\circ} \mathrm{C}$. equals. The second factor reduces the height of this to millimetres. The third gives the result at $20^{\circ} \mathrm{C}$., supposing the vapour to follow Boyle's Law. The fourth is the part of a litre there was to be considered.

The expression for the mean freo path in these surfacevapours is then

$$
{ }_{2} \mathrm{~L}=\cdot 647 \cdot \frac{\cdot 80357}{13 \cdot 579} \cdot 100 \cdot \frac{18 \cdot 495}{760} \cdot \frac{23 \cdot 379}{100} \cdot \frac{1}{18 \cdot 495},
$$

$r=\cdot 647$.

This gives $\mathrm{L}=\cdot 0000024$ millim.

If the law, according to which the density of the vapours varies with the depth, were known, the free path of a molecule in a gas at the ordinary pressure could be found.

Physical Laboratory, Harvard College,

Cambridge, U. S. A., Jan. 27, 1880.

XXV. Crystallographic Notes. By L. Fletcher, M.A., Fellow of University College, Oxford, Assistant in the Mineralogical Department, British Museum*.

[Plate V.]

\section{Copper.}

$7 \mathrm{THE}^{\mathrm{HE}}$ following forms have already been described as occur1 ring on native copper:-
$(100)$
(110)
(1 111$)$
(2 10$)$
$(520)$
(3 111$)$
(4 12)
(18 10 5).

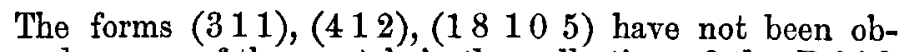
served on any of the crystals in the collection of the British Museum. The form (31 1 ) was described by Roset as occurring on some rude crystals from Nijni-Tagilsk: the crystals

* Communicated by the Crystallological Society.

+ Reise nach dem Ural, von G. Rose, vol, i. p. 313 (1837). 